\title{
1 A novel intranasal administration adenoviral vector-based 2 platform for rapid COVID-19 vaccine development
}

3 Yifei Yuan ${ }^{1}$, Xing $\mathrm{Gao}^{2}$, Fengfeng $\mathrm{Ni}^{3,4}$, Wenbo Xie ${ }^{1}$, Wenbin $\mathrm{Fu}^{2}$, Gaoxia Zhang ${ }^{2}$,

4 Huimin $\mathrm{Hu}^{3,4}$, Yuncheng $\mathrm{Li}^{3,4}$, Qinxue $\mathrm{Hu}^{3,5}$, Chuanmengyuan Huang ${ }^{1}$, Bo Liu ${ }^{1}$, Yalan

$5 \quad$ Liu $^{3 *}$, Qiong Shen ${ }^{2 *}$, Min Liang ${ }^{1 *}$

6

$7 \quad{ }^{1}$ GeneSail Biotech (Shanghai) Co., Ltd., Shanghai, China

$8 \quad{ }^{2}$ Shanghai Bovax Biotechnology Co., Ltd., Shanghai, China

$9{ }^{3}$ State Key Laboratory of Virology, Wuhan Institute of Virology, Center for Biosafety

10 Mega-Science, Chinese Academy of Sciences, Wuhan, China

$11{ }^{4}$ Savaid Medical School, University of Chinese Academy of Sciences, Beijing, China

$12{ }^{5}$ Institute for Infection and Immunity, St George's, University of London, London, 13 UK

14

15 Correspondence: Yalan Liu (liuyl@wh.iov.cn), Qiong Shen

16 (Shenqiong@bovax.com.cn) or Min Liang (liangmin@genesailbiotech.com)

17

18 These authors contributed equally: Yifei Yuan, Xing Gao, Fengfeng Ni

19

20 


\section{ABSTRACT}

22 The coronavirus SARS-CoV-2 has a severe impact on global public health, and the

23 emerging variants threaten the efficacy of the circulating vaccines. Here, we report

24 that a single vaccination with a non-replicated Chimpanzee adenovirus-based vaccine

25 against the SARS-CoV-2 B.1.617.2 variant (JS1-delta) elicits potent humoral, cellular

26 and mucosal immunity in mice. Additionally, a single intranasal administration of

27 JS1-delta provides sufficient protection against B.1.617.2 challenge in mice. This

28 study indicates that Chimpanzee adenovirus type 3 (ChAd3) derived vector represents

29 a promising platform for antiviral vaccine development against respiratory infections,

30 and that JS1-delta is worth further investigation in human clinical trials. 
32

33

34

\section{RESULTS}

\section{2} examined.

\section{INTRODUCTION}

The global coronavirus disease 2019 (COVID-19) pandemic caused by SARS-CoV-2 has severe effects on public health. As of 16 February 2022, there have been over 412 million laboratory-confirmed COVID-19 cases and more than 5.8 million conformed deaths. ${ }^{1}$ Although the currently used vaccines have been demonstrated to reduce severe disease and death effectively, the constantly emerging SARS-CoV-2 variants, such as B.1.617.2 (Delta) and B.1.1.529 (Omicron) which have spread quickly and widely around the world, pose serious threats to existing immunologic barrier by compromising the neutralization capability of antibodies and causing breakthrough infection. $^{2-4}$ Therefore, rapid response systems for developing vaccines against the pandemic variants should be urgently needed.

Here, we reported the study of a non-replicated Chimpanzee adenovirus-based vaccine against the SARS-CoV-2 delta variant, which is one of the variants of concern (VOC). We evaluated the humoral and cellular immune responses elicited by the candidate vaccine in mice. The induction of mucosal immunity and protective efficacy of the candidate vaccine against B.1.617.2 challenge in mice have also been

\section{Construction and identification of JS1-delta}


53 To develop a prophylactic vaccine against the SARS-CoV-2 delta variant, we

54 constructed a recombinant replication-incompetent adenovirus carrying optimized

55 S-coding sequence, named JS1-delta. Chimpanzee adenovirus type 3 (ChAd3,

56 GenBank ID: CS138463) was chosen to delete E1 region (nt 589-3544) and E3 region

57 (nt 28310-32653). Meanwhile, ChAd3 E4 region (nt 34698-37314) was replaced by

58 the E4 region of human adenovirus type 5 (hAd5, GenBank ID: AY339865, nt

59 32909-35522). Two endonucleases, I-Ceu $\square$ and PI-Sce $\square$, were inserted into the

60 original ChAd3 E1 region. This recombinant ChAd3 vector was named JS1 (Fig. 1a).

61 The S-coding sequence from B.1.617.2 was optimized by altering the codon usages to

62 increase its antigen expression in mammalian cells. ${ }^{5,6}$ At the same time, the furin

63 cleavage site mutations (R682S, R685G) and proline substitutions (K986P, V987P)

64 were introduced in the sequence to stabilize the $\mathrm{S}$ protein and prevent syncytium

65 formation. ${ }^{7}$ Then, with the addition of CMV promoter and BGH poly(A) signal, the

66 optimized S-coding sequence was inserted between I-Ceu $\square$ and PI-Sce $\square$ in JS1 (Fig.

67 1b). As shown in Fig. 1c, JS1-delta was successfully rescued and multiplied in

68 HEK293 cells, resulting in efficient expression of S protein.

70 JS1-delta induces potent humoral and cellular immune responses in mice

71 To assess the immunogenicity of JS1-delta, six-week-old female BALB/c mice ( $\mathrm{n}=5$

72 per group) received a single immunization of $5 \times 10^{7}$ virus particles (VP) (low dose),

$735 \times 10^{8}$ VP (middle dose), $5 \times 10^{9} \mathrm{VP}$ (high dose) of JS1-delta or $5 \times 10^{9} \mathrm{VP}$ of the 
74 control vector JS1 via the intramuscular (IM) or intranasal (IN) route at day 0. The

75 titers of delta-S1-specific IgG, neutralizing antibody (NAb) and cellular immune

76 responses were detected in each group. The serum IgG titers were detectable at week

772 and stayed at a high level until week 8 (Fig. 2a). There were higher IgG titers in all

78 three IM groups than in the relative IN groups at week 2, but at week 8 the IN groups

79 showed no significant difference with the IM groups. Similar to the IgG titers, the

80 B.1.617.2 pseudovirus NAb (PNAb) titers were detectable at week 2 and stayed

81 steady until week 8 (Fig. 2b). At week 2, there was no difference between the IN and

82 IM groups, but the PNAb titer in the low-dose IN group was slightly higher than that

83 in the low-dose IM group at week 8, without difference in the middle- and high-dose

84 groups at the same time. Moreover, both the high-dose IN and IM groups elicited

85 potent IFN $\gamma$ and IL-2 responses in splenic lymphocytes at week 8 (Fig. 2c).

JS1-delta induces effective mucosal immune responses in hACE2 transgenic mice

To further study the capacity of JS1-delta for mucosal immunity induction,

90 per group) were immunized intranasally with $5 \times 10^{8}$ VP (low dose) and $5 \times 10^{9}$ VP

91 (high dose) of JS1-delta or the same volume of saline as a control at day 0. The

92 delta-S-specific IgA titers were examined in each group at week 4 post vaccination.

93 As shown in Fig. 2d and 2e, both low- and high-dose groups elicited effective

94 S-specific IgA expressions in the bronchoalveolar lavage fluids (BALFs) and the 
95 saliva of mice. Meanwhile, the cross-protections of JS1-delta to SARS-CoV-2

96 prototype, B.1.1.7 (Alpha), B.1.351 (Beta) and B.1.617.2 variants were also evaluated.

97 As shown in Fig. 2f, the high-dose group showed effective protection to all four

98 SARS-CoV-2 strains at week 4. But compared with those against the delta variant, the

99 NAb titers against other three variants decreased by about 4 6-fold.

100

$101 \quad$ JS1-delta protects mice from B.1.617.2 infection

102 To determine the protective efficacy of JS1-delta through the mucosal vaccination,

103 4-week-old female H11-K18-hACE2 mice were immunized with $5 \times 10^{8} \mathrm{VP}$ (low dose)

104 and $5 \times 10^{9} \mathrm{VP}$ (high dose) of JS1-delta by the IN route at day 0 . Then, mice were

105 challenged with $1 \times 10^{3}$ PFU of B.1.617.2 virus by nasal drop at day 28 (Fig. 3a). The

106 titers of delta-S-specific serum IgG and NAbs were tested at day -2 and day 26, and

107 all vaccinated mice showed high levels of serum IgG and NAbs, which were not

108 detectable in control animals (Fig. 3b, c). Whereafter, we monitored the weight

109 change and the survival rate in each group. The evaluating indicators kept normal in

110 all groups until day 3 post-infection ( $3 \mathrm{dpi}$ ), but the infected mice without

111 immunization (saline group) lost weight obviously from 4 dpi and all died at 5 dpi,

112 while other groups remained stable and stayed alive (Fig. 3d, e). Subsequently, mice

113 were euthanized at 6 dpi to detect the viral load in lung tissue and for

114 immunofluorescence or pathological examination. As expected, only the saline group

115 exhibited high level of viral RNA copies, displayed lots of SARS-CoV-2 N protein 
116 positive cells and showed severe histopathological changes in lung tissue, which were

117 not detectable in vaccinated animals (Fig. 3f, g). Taken together, all these results

118 indicated that both low- and high-dose JS1-delta can confer sufficient protection

119 against B.1.617.2 infection in hACE2 transgenic mice.

120

\section{DISCUSSION}

122 Multiple reports have found that the SARS-CoV-2 variants can reduce the

123 neutralization capability of monoclonal antibodies (mAbs) and convalescent plasma,

124 and can resist antibody-mediated immunity elicited by vaccines. ${ }^{4,8-14}$ As stated by the

125 WHO Technical Advisory Group on COVID-19 Vaccine Composition

126 (TAG-CO-VAC), considering supply and demand of the available vaccines, along

127 with evolution of the virus, repeated booster doses of the original vaccine composition

128 is unlikely to be appropriate or sustainable. ${ }^{15}$ Based on the reality that the new

129 variants emerge constantly, it is not enough for vaccines to just reduce hospitalization

130 and mortality, but should be more effective in preventing infection and transmission.

131 In our study, JS1-delta has been demonstrated to protect mice from B.1.617.2

132 infection sufficiently (Fig. 3). However, its cross-protection against other variants is

133 attenuated (Fig. 2d), which is consistent with another SARS-CoV-2 candidate vaccine

134 JS1-beta (Fig. S1e). These results and reports suggest that a monovalent vaccine used

135 independently may be difficult to handle the complex epidemic situation. Moreover, it

136 is reported that the heterologous prime-boost strategy with COVID-19 vaccines from 
137 diverse platforms could be more effective than using a homologous booster. ${ }^{16,17}$

138 Therefore, using heterologous boosters or developing multivalent vaccines containing

139 various SARS-CoV-2 variants compositions may be more efficient and practical to

140 fight against the virus.

142 Replication deficient recombinant adenoviral vectors possess great immunogenicity

143 and can elicit potent cellular immune responses in preclinical and clinical researches,

144 in which hAd5 is the most comprehensively studied. ${ }^{18-22}$ However, hAd5 shows high

145 seroprevalence in global populations due to natural infection, especially in the

146 developing world, and high level of the pre-existing immunity could result in

147 decreased efficacy of the hAd5-based vaccines. ${ }^{18,19}$ Meanwhile, considering that

148 HEK293 remains a major producer cell line for adenovirus development and

149 manufacturing, replication-competent adenovirus (RCA) contamination is a

150 significant trouble for large-scale production and clinical application of the

151 hAd5-derived vectors. ${ }^{23,24}$ To circumvent these limitations, considerable efforts have

152 been made to develop new adenoviral vectors from lower seroprevalence hAds (such

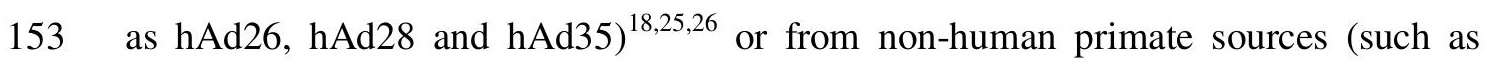

154 ChAdY25 and ChAd63) $)^{27,28}$. ChAd3 is one of the most promising candidates and

155 shows well safety and tolerance in clinical trials targeting hepatitis $\mathrm{C}$ virus and Ebola

156 virus. $^{29-32}$ In this study, we have demonstrated for the first time, to our knowledge,

157 that a ChAd3-derived SARS-CoV-2 vaccine can induce potent humoral and cellular 
158 immune responses and completely protect mice from B.1.617.2 challenge. At the

159 same time, another vaccine candidate JS1-beta targeting B.1.351 shows favorable

160 immunogenicity in mice (Fig. S1), and JS1-omicron targeting B.1.1.529 is also under

161 testing. The longevity of antigen-specific immune responses and the influence of

162 anti-vector immunity induced by the vaccines, along with the cooperation with

163 different boosters, will be further investigated.

164

165 Recently, mucosal vaccines against SARS-CoV-2 have raised increasing interests due

166 to their important advantage in preventing virus replication in the upper respiratory

167 tracts, which is essential to interrupt pulmonary infection and person to person

168 transmission. ${ }^{33-36}$ Compared with IM injection, the IN vaccination can elicit not only

169 comparable systemic immunity but also additional pathogen-specific mucosal

170 immunity, resulting in a first line of defense against the virus invasion. ${ }^{34-36}$ In our

171 study, IN vaccination of JS1-delta induces S-specific IgA expressions efficiently in

172 both BALFs and saliva of the hACE2 transgenic mice (Fig. 2e, f). Using

173 SARS-CoV-2 as a model, our findings both in vitro and in vivo highlight that the

174 non-replicating ChAd3-derived vector administrated in the IN route represents a

175 powerful platform for rapid vaccine development against respiratory virus infections.

176

177 MATERIALS AND METHODS

178 Construction of JS1-delta 
179 The chemosynthetic JS1 genome was cyclized by the additional pUC57 backbone and

180 was linearized by a double enzyme digestion with I-Ceu $\square$ and PI-Sce $\square$. Then, the

181 chemosynthetic delta-S sequence was inserted into JS1 genome by homologous

182 recombination, and JS1-delta genome was released from the backbone by the

183 digestion with $\mathrm{P} a c$ $\square$. Finally, HEK293 (ATCC) cells were transfected with linear

184 JS1-delta genome to rescue the recombinant virus.

185

186 Animals

187 The experiments involving animals were approved by and carried out in accordance

188 with the guidelines of the Institutional Experimental Animal Welfare and Ethics

189 Committee. Specific pathogen-free female BALB/c mice were obtained from Charles

190 River Laboratories. Specific pathogen-free female H11-K18-hACE2 mice were

191 obtained from GemPharmatech.

192

193 Purification of SARS-CoV-2 delta-S protein

194 SARS-CoV-2 delta-S protein (1-1141aa) with a C-terminal 6xHis tag was expressed

195 in HEK293 cells. In brief, adherent HEK293 cells were transfected with recombinant

196 pCDNA3.1-delta S-His (1-1141) using PEI and incubated in $37^{\circ} \mathrm{C}, 5 \% \mathrm{CO}_{2}$ for $72 \mathrm{~h}$.

197 The supernatant was harvested by centrifugation and purified by Ni-NTA affinity

198 chromatography.

199 
200

201 The antigen expression in JS1-delta infected HEK293 cells was examined by SARS-CoV2 (2019-nCoV) Spike RBD ELISA KIT (SinoBiological) following the manufacturer's instruction. To measure the titers of SARS-CoV-2-specific IgG or

204 IgA, 96-well clear polystyrene microplates (Corning) were coated with delta-S1 205 protein (GenScript) or purified delta-S protein and incubated at $4{ }^{\circ} \mathrm{C}$ overnight. After 206 washes for 2-4 times, the plates were blocked at $37{ }^{\circ} \mathrm{C}$ for $1 \mathrm{~h}$ with $5 \%$ skim milk in 207 PBS and washed again with PBST. The plates were then added with $100 \mu \mathrm{L}$ serially 208 diluted sera or BALFs or saliva and incubated at $37{ }^{\circ} \mathrm{C}$ for $1 \mathrm{~h}$. Following washes, 209 HRP-conjugated Affinipure Goat Anti-Mouse IgG (H+L) (Proteintech, 1:5000 210 dilution) or Goat Anti-Mouse IgA-HRP (SouthernBiotech, 1:5000 dilution) was added

211 to the plates and incubated for $1 \mathrm{~h}$ at $37{ }^{\circ} \mathrm{C}$. The assays were developed with TMB

212 substrate (Sigma), stopped by the addition of $2 \mathrm{M} \mathrm{H}_{2} \mathrm{SO}_{4}$ and were measured at 450 $213 \mathrm{~nm} / 630 \mathrm{~nm}$. The endpoint titre was defined as the highest dilution yielding an 214 absorbance $>2$-fold over the values of negative control.

216 Neutralization assay

217 To test SARS-CoV-2 PNAbs, serial dilutions of heat-inactivated sera were mixed with 218 B.1.617.2 pseudoviruse (Beijing Tiantan Pharmacy Biological Research \& 219 Development Company), incubated for $1 \mathrm{~h}$ at $37{ }^{\circ} \mathrm{C}$ and added to ACE2-293T 220 (YEASEN) cells in duplicate in 96-well microplate. Cells were lysed $24 \mathrm{~h}$ later and 
221 luciferase activity was measured. The neutralization titers were calculated as the

222 serum dilution at which the luciferase activity reduced by $50 \%$ compared with the

223 virus-only wells.

224

225 To test SARS-CoV-2 NAbs, serial dilutions of heat-inactivated sera were incubated

226 with $70 \mathrm{PFU}$ of the indicated variants (isolated and stored by Wuhan Institute of

227 Virology, CAS) at $37{ }^{\circ} \mathrm{C}$ for $1 \mathrm{~h}$. The mixtures were added to pre-plated Vero E6

228 (ATCC) cell monolayers in 96-well microplate and cultured for 3-4 day. NAb titres

229 were calculated by Reed Muench method to estimate the serum dilution required for a

$23050 \%$ CPE reduction.

232 ELISpot

233 SARS-CoV-2-specific cellular immune responses were assessed by the IFN $\gamma$ and IL-2

234 ELISpot Kit (Cellular Technology Limited) following the manufacturer's instructions.

235 In brief, $1 \times 10^{6}$ of mice splenic lymphocytes were stimulated with delta-S protein in a

236 pre-coated ELISpot plate for $16 \mathrm{~h}$ at $37^{\circ} \mathrm{C}$. After being washed twice, the plates were

237 added with Anti-murin IFN $\gamma$ Detetion Solution or Anti-murin IL-2 Detetion Solution

238 and incubated at room temperature for $2 \mathrm{~h}$. The plates were then added with Tertiary

239 Solution and Blue Developer Solution. Finally, the spots were counted with

240 ImmunoSpot Analyzer (CTL S6 uNVERSAL). 
242

243 Viral RNA was extracted using TRI Reagent (Sigma). Reverse transcription was

244 performed using HiScript II Q RT SuperMix for qPCR (Vazyme). Subsequently, $5 \mu$ l

245 cDNA was added into a $25 \mu \mathrm{l}$ qRT-PCR reaction containing ChamQ SYBR qPCR

246 Master Mix (Vazyme). The primers designed to target the nucleocapsid protein of

247 SARS-CoV-2 were (forward) 5'-GGG GAA CTT CTC CTG CTA GAA T-3' and

248 (reverse) 5'-CAG ACA TTT TGC TCT CAA GCT G-3'. The samples were run in

249 triplicate on an ABI 7900 Real-Time System (Applied Biosystems, Thermo). The

250 following cycling conditions were performed: 1 cycle of $50{ }^{\circ} \mathrm{C}$ for 2 min, 1 cycle of

$25195{ }^{\circ} \mathrm{C}$ for $10 \mathrm{~min}$, and 40 cycles of $95^{\circ} \mathrm{C}$ for $15 \mathrm{sec}$ and $58{ }^{\circ} \mathrm{C}$ for $1 \mathrm{~min}$. Finally, the

252 amount of viral RNA was normalized to the standard curve from a plasmid containing

253 the full-length SARS-CoV-2 $N$ gene.

$255 \quad$ IF and H\&E

256 The lung tissues were immersed in 10\% neutral buffered formalin (Sigma) for $24 \mathrm{~h}$.

257 After the formalin fixation, the tissues were placed in $70 \%$ ethanol (Merck) and

258 subsequently embedded with paraffin. Tissue sections (5- $\mu \mathrm{m}$ thick) were prepared for

259 H\&E staining or immunofluorescence staining using SARS-CoV/SARS-CoV-2

260 Nucleocapsid Antibody (SinoBiological). Images were collected under a Pannoramic

261 MIDI system (3DHISTECH, Thermo) using Pannoramic scanner software and 262 analyzed by Image J (NIH). 
264 Statistical analysis

265 Data are presented as the mean \pm standard error of the mean. Statistics were

266 performed using the nonparametric Mann-Whitney U test or the Student $t$ test. $\mathrm{P}<0.05$

267 was considered statistically significant $(* \mathrm{p}<0.05, * * \mathrm{p}<0.01)$.

\section{ACKNOWLEDGEMENTS}

270 We are particularly grateful to Prof. Bing Sun and Dr. Xiaoyu Sun (Center for

271 Excellence in Molecular Cell Science, CAS) for the technical assistance in $\operatorname{IgG}$

272 ELISA and PNAb assay.

273

274 AUTHOR CONTRIBUTIONS

275 M.L. conceived this project. Y.Y. designed the study and wrote the manuscript with 276 input from all authors. W.X., C.H. and B.L. constructed the virus and performed the 277 experiment in BALB/c mice. W.F. performed the protein purification and ELISpot. 278 Q.S. designed the challenge study. F.N., H.H., Y.Li, Q.H. and Y.Liu evaluated the 279 efficacy of JS1-delta in B.1.617.2 infected transgenic hACE2 mice. X.G. and G.Z. 280 coordinated the project.

282 Competing interests: The authors declare no competing interests. 


\section{REFERENCES}

2851 WHO. Coronavirus disease (COVID-19) situation report. (2022).

2862 Hacisuleyman, E. et al. Vaccine Breakthrough Infections with SARS-CoV-2

287 Variants. $N$ Engl J Med 384, 2212-2218, doi:10.1056/NEJMoa2105000

$288 \quad(2021)$.

2893 Ma, C. et al. Mild Breakthrough Infection in a Healthcare Professional

290 Working in the Isolation Area of a Hospital Designated for Treating

291 COVID-19 Patients - Shaanxi Province, China, March, 2021. China CDC

292 Wkly 3, 397-400, doi:10.46234/ccdcw2021.094 (2021).

2934 Tian, D., Sun, Y., Zhou, J. \& Ye, Q. The global epidemic of SARS-CoV-2

294 variants and their mutational immune escape. J Med Virol, 295 doi:10.1002/jmv.27376 (2021).

2965 Liu, J. et al. BNT162b2-elicited neutralization of B.1.617 and other SARS-CoV-2 variants. $\quad$ Nature 273-275, 298 doi:10.1038/s41586-021-03693-y (2021).

2996 Planas, D. et al. Reduced sensitivity of SARS-CoV-2 variant Delta to antibody 300 neutralization. Nature 596, 276-280, doi:10.1038/s41586-021-03777-9 (2021).

3017 Bos, R. et al. Ad26 vector-based COVID-19 vaccine encoding a 302 prefusion-stabilized SARS-CoV-2 Spike immunogen induces potent humoral 303 and cellular immune responses. NPJ Vaccines 5, 91, 304 doi:10.1038/s41541-020-00243-x (2020). 
3058 Lustig, Y. et al. Neutralising capacity against Delta (B.1.617.2) and other

306 variants of concern following Comirnaty (BNT162b2, BioNTech/Pfizer)

307 vaccination in health care workers, Israel. Euro Surveill 26,

308 doi:10.2807/1560-7917.Es.2021.26.26.2100557 (2021).

3099 Shen, X. et al. SARS-CoV-2 variant B.1.1.7 is susceptible to neutralizing

310 antibodies elicited by ancestral spike vaccines. Cell Host Microbe 29,

311 529-539.e523, doi:10.1016/j.chom.2021.03.002 (2021).

31210 Cao, Y. et al. Omicron escapes the majority of existing SARS-CoV-2

313 neutralizing antibodies. Nature, doi:10.1038/s41586-021-04385-3 (2021).

31411 Cele, S. et al. Omicron extensively but incompletely escapes Pfizer

315 BNT162b2 neutralization. Nature, doi:10.1038/s41586-021-04387-1 (2021).

31612 Li, Q. et al. SARS-CoV-2 501Y.V2 variants lack higher infectivity but do have

317 immune escape. Cell 184, 2362-2371.e2369, doi:10.1016/j.cell.2021.02.042

$318 \quad$ (2021).

31913 Liu, C. et al. Reduced neutralization of SARS-CoV-2 B.1.617 by vaccine and

320 convalescent $\quad$ serum. Cell $\quad \mathbf{1 8 4}, \quad$ 4220-4236.e4213,

321 doi:10.1016/j.cell.2021.06.020 (2021).

32214 Cao, Y. et al. Humoral immune response to circulating SARS-CoV-2 variants

323 elicited by inactivated and RBD-subunit vaccines. Cell Res 31, 732-741,

324 doi:10.1038/s41422-021-00514-9 (2021).

32515 WHO. Interim Statement on COVID-19 vaccines in the context of the 
326 circulation of the Omicron SARS-CoV-2 Variant from the WHO Technical

327 Advisory Group on COVID-19 Vaccine Composition (TAG-CO-VAC).

$328 \quad$ (2022).

32916 Liu, X. et al. Safety and immunogenicity of heterologous versus homologous

330 prime-boost schedules with an adenoviral vectored and mRNA COVID-19

331 vaccine (Com-COV): a single-blind, randomised, non-inferiority trial. Lancet

332 398, 856-869, doi:10.1016/s0140-6736(21)01694-9 (2021).

33317 Zhang, J. et al. Boosting with heterologous vaccines effectively improves

334 protective immune responses of the inactivated SARS-CoV-2 vaccine. Emerg

335 Microbes Infect 10, 1598-1608, doi:10.1080/22221751.2021.1957401 (2021).

33618 Abbink, P. et al. Comparative seroprevalence and immunogenicity of six rare

337 serotype recombinant adenovirus vaccine vectors from subgroups B and D. $J$

$338 \quad$ Virol 81, 4654-4663, doi:10.1128/jvi.02696-06 (2007).

33919 Quinn, K. M. et al. Comparative analysis of the magnitude, quality, phenotype,

340 and protective capacity of simian immunodeficiency virus gag-specific CD8+

341 T cells following human-, simian-, and chimpanzee-derived recombinant

342 adenoviral vector immunization. J Immunol 190, 2720-2735,

343 doi:10.4049/jimmunol.1202861 (2013).

34420 Sullivan, N. J. et al. Accelerated vaccination for Ebola virus haemorrhagic

345 fever in non-human primates. Nature 424, 681-684, doi:10.1038/nature01876

$346 \quad$ (2003). 
34721 Bassett, J. D., Swift, S. L. \& Bramson, J. L. Optimizing vaccine-induced

348 CD8(+) T-cell immunity: focus on recombinant adenovirus vectors. Expert

349 Rev Vaccines 10, 1307-1319, doi:10.1586/erv.11.88 (2011).

35022 Catanzaro, A. T. et al. Phase 1 safety and immunogenicity evaluation of a 351 multiclade HIV-1 candidate vaccine delivered by a replication-defective 352 recombinant adenovirus vector. $J$ Infect Dis 194, 1638-1649, 353 doi:10.1086/509258 (2006).

35423 Kovesdi, I. \& Hedley, S. J. Adenoviral producer cells. Viruses 2, 1681-1703, 355 doi:10.3390/v2081681 (2010).

35624 Lusky, M. Good manufacturing practice production of adenoviral vectors for 357 clinical trials. Hum Gene Ther 16, 281-291, doi:10.1089/hum.2005.16.281 $358 \quad(2005)$

35925 Kahl, C. A. et al. Potent immune responses and in vitro pro-inflammatory 360 cytokine suppression by a novel adenovirus vaccine vector based on rare 361 human $\quad$ serotype 28 Vaccine $28, \quad 5691-5702$, 362 doi:10.1016/j.vaccine.2010.06.050 (2010).

36326 Vogels, R. et al. Replication-deficient human adenovirus type 35 vectors for 364 gene transfer and vaccination: efficient human cell infection and bypass of 365 preexisting adenovirus immunity. $J$ Virol 77, 8263-8271, 366 doi:10.1128/jvi.77.15.8263-8271.2003 (2003).

36727 Dicks, M. D. et al. A novel chimpanzee adenovirus vector with low human 
368 seroprevalence: improved systems for vector derivation and comparative

369 immunogenicity. PLoS One 7, e40385, doi:10.1371/journal.pone.0040385

$370 \quad$ (2012).

37128 O'Hara, G. A. et al. Clinical assessment of a recombinant simian adenovirus

372 ChAd63: a potent new vaccine vector. J Infect Dis 205, 772-781,

373 doi:10.1093/infdis/jir850 (2012).

37429 Barnes, E. et al. Novel adenovirus-based vaccines induce broad and sustained

$375 \mathrm{~T}$ cell responses to $\mathrm{HCV}$ in man. Sci Transl Med 4, 115ra111,

376 doi:10.1126/scitranslmed.3003155 (2012).

37730 Kelly, C. et al. Chronic hepatitis C viral infection subverts vaccine-induced

378 T-cell immunity in humans. Hepatology 63, 1455-1470,

379 doi:10.1002/hep.28294 (2016).

38031 De Santis, O. et al. Safety and immunogenicity of a chimpanzee 381 adenovirus-vectored Ebola vaccine in healthy adults: a randomised, 382 double-blind, placebo-controlled, dose-finding, phase 1/2a study. Lancet Infect 383 Dis 16, 311-320, doi:10.1016/s1473-3099(15)00486-7 (2016).

38432 Ledgerwood, J. E. et al. Chimpanzee Adenovirus Vector Ebola Vaccine. $N$ 385 Engl J Med 376, 928-938, doi:10.1056/NEJMoa1410863 (2017).

38633 Lavelle, E. C. \& Ward, R. W. Mucosal vaccines - fortifying the frontiers. Nat 387 Rev Immunol, 1-15, doi:10.1038/s41577-021-00583-2 (2021).

38834 Feng, L. et al. An adenovirus-vectored COVID-19 vaccine confers protection 
389 from SARS-COV-2 challenge in rhesus macaques. Nat Commun 11, 4207,

390 doi:10.1038/s41467-020-18077-5 (2020).

39135 Hassan, A. O. et al. A Single-Dose Intranasal ChAd Vaccine Protects Upper

392 and Lower Respiratory Tracts against SARS-CoV-2. Cell 183, 169-184.e113,

393 doi:10.1016/j.cell.2020.08.026 (2020).

$39436 \mathrm{Wu}$, S. et al. A single dose of an adenovirus-vectored vaccine provides

395 protection against SARS-CoV-2 challenge. Nat Commun 11, 4081,

396 doi:10.1038/s41467-020-17972-1 (2020). 


\section{FIGURE LEGENDS}

Fig.1 Construction and identification of JS1-delta. a, b Schematic of JS1 and

JS1-delta genome. CMV: cytomegalovirus promoter. RBD: receptor-binding domain of SARS-CoV-2 spike protein. BGH: bovine growth hormone poly(A) signal. c HEK293 cells were infected with JS1-delta and then cultured for 48 hours. The cell supernatant was collected to detect the expression of S protein by ELISA.

Fig.2 The immunogenicity of JS1-delta in mice. a-c 6-week-old BALB/c mice ( $\mathrm{n}=5$ per group) were vaccinated with JS1-delta or the control vector (JS1) using the indicated dosage by the IN or IM route at day 0 . The titers of S1-specific IgG, B.1.617.2 PNAb and the levels of cellular immune responses at week 8 were detected by ELISA, luciferase assay and ELISpot, respectively. d-f 4-week-old H11-K18-hACE2 mice ( $\mathrm{n}=6$ per group) were immunized intranasally with $5 \times 108$ VP (low dose) and $5 \times 109 \mathrm{VP}$ (high dose) of JS1-delta or the same volume of saline at day 0. At week 4, the S-specific IgA titers in BALFs or saliva were examined by ELISA. The NAb titers against the indicated SARS-COV-2 strains were detected by CPE calculation.

Fig.3 JS1-delta protects mice from B.1.617.2 infection. a Schematic of experimental strategy and grouping. b, c 4-week-old H11-K18-hACE2 mice were immunized intranasally with $5 \times 108$ VP (low dose, n=10) and 5×109 VP (high dose, 
$\mathrm{n}=10)$ of JS1-delta or the same volume of saline $(\mathrm{n}=6)$ at day 0 . Then, the mice were challenged with $1 \times 103$ PFU of B.1.617.2 virus in a volume of $50 \mu \mathrm{L}$ by nasal drop at day 28. Serums were collected at day -2 and day 26 , and the titers of S-specific IgG and B.1.617.2 NAb were detected by ELISA and CPE calculation. d The weight change was monitored every week after vaccination and every day after infection. $\mathbf{e}$ The survival rate was monitored every day after infection. f, g The mice in saline group died at $5 \mathrm{dpi}$, the others were sacrificed at $6 \mathrm{dpi}$. The viral load in lung tissue was detected by qPCR. SARS-COV-2 $\mathrm{N}$ protein expression and histopathological examinations in lungs were examined by immunofluorescence (IF) and hematoxylin-eosin staining (H\&E). 
Fig.1

a I-Ceu I PI-Sce I

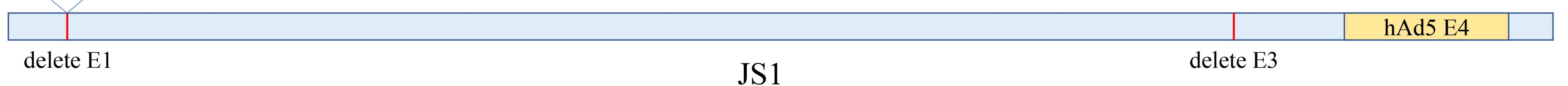

b I-Ceu I PI-Sce I
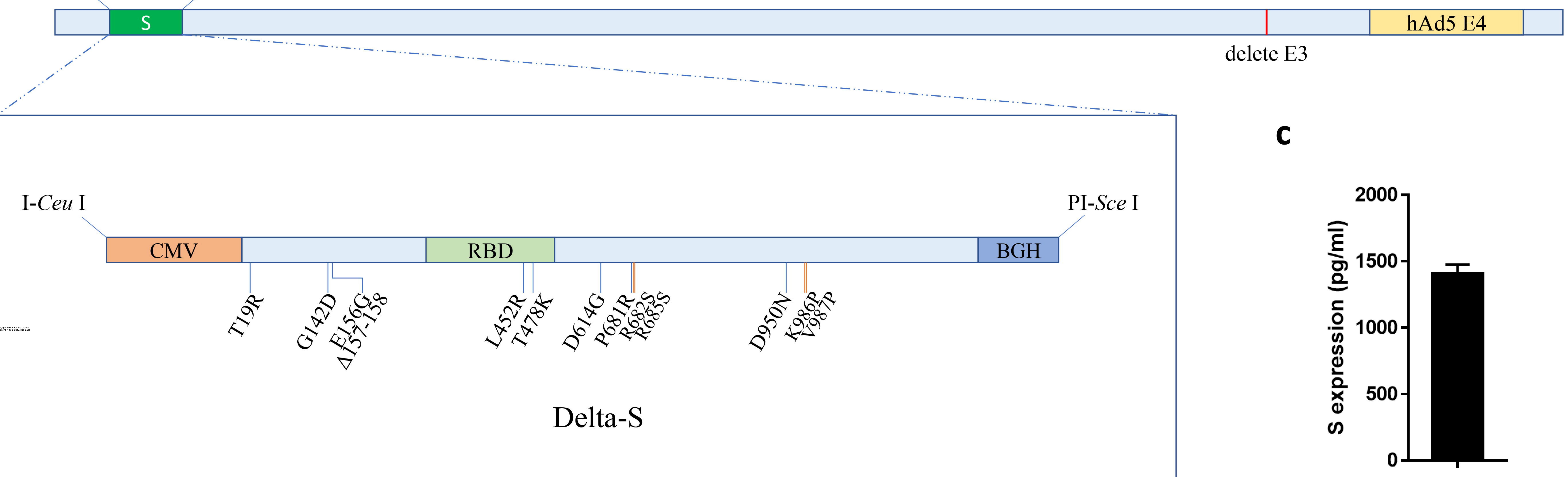

C

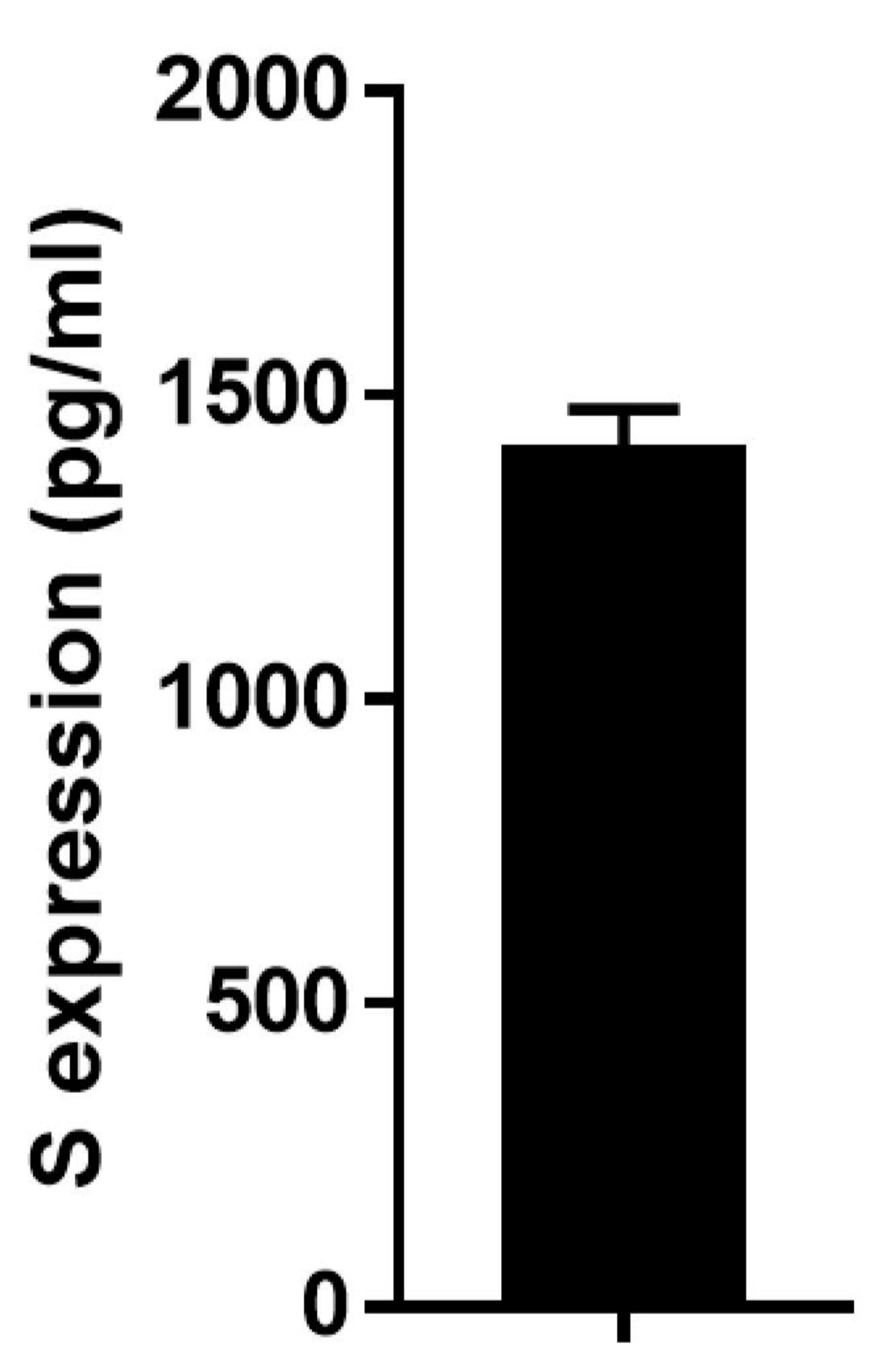


Fig.2

a

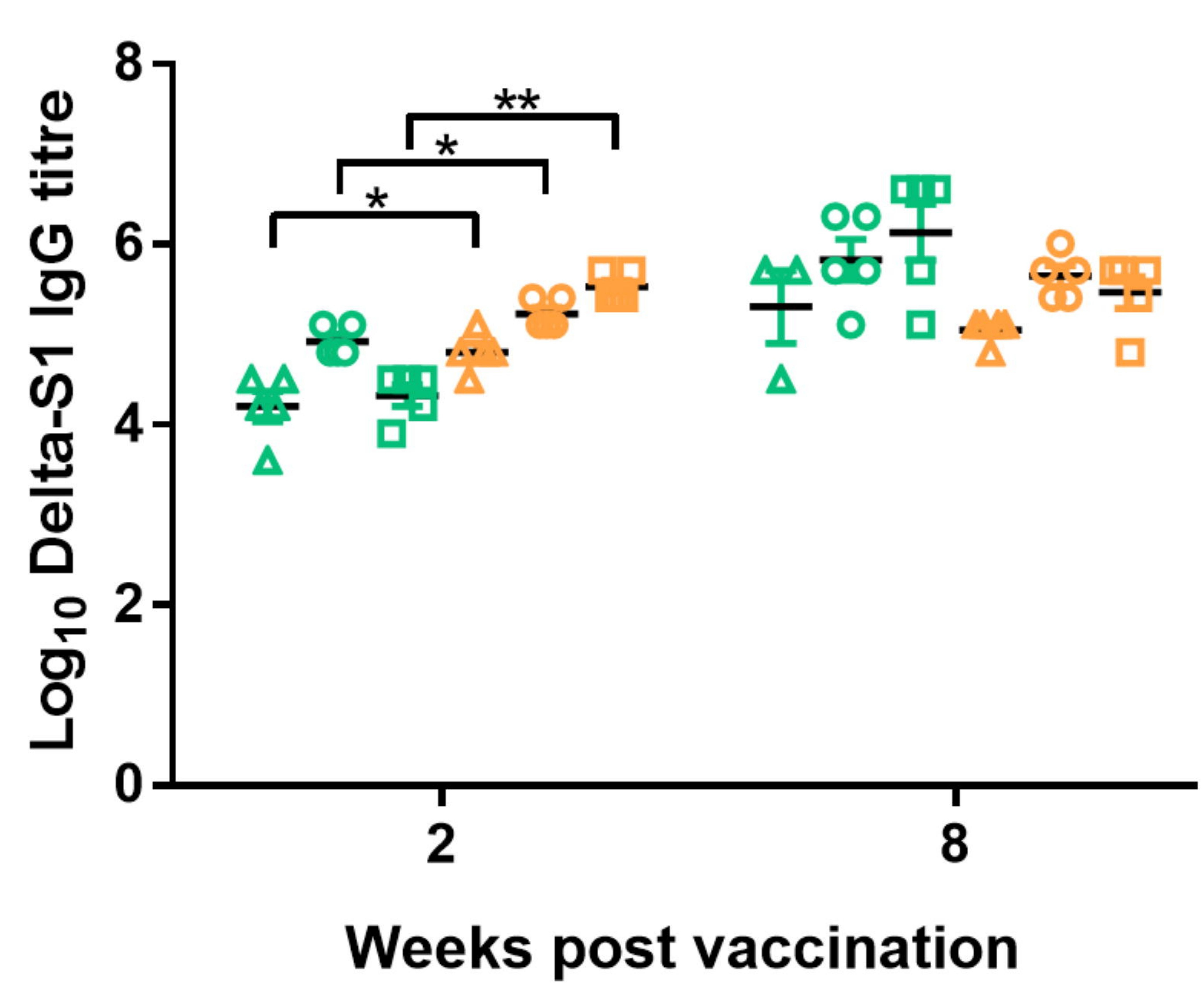

b

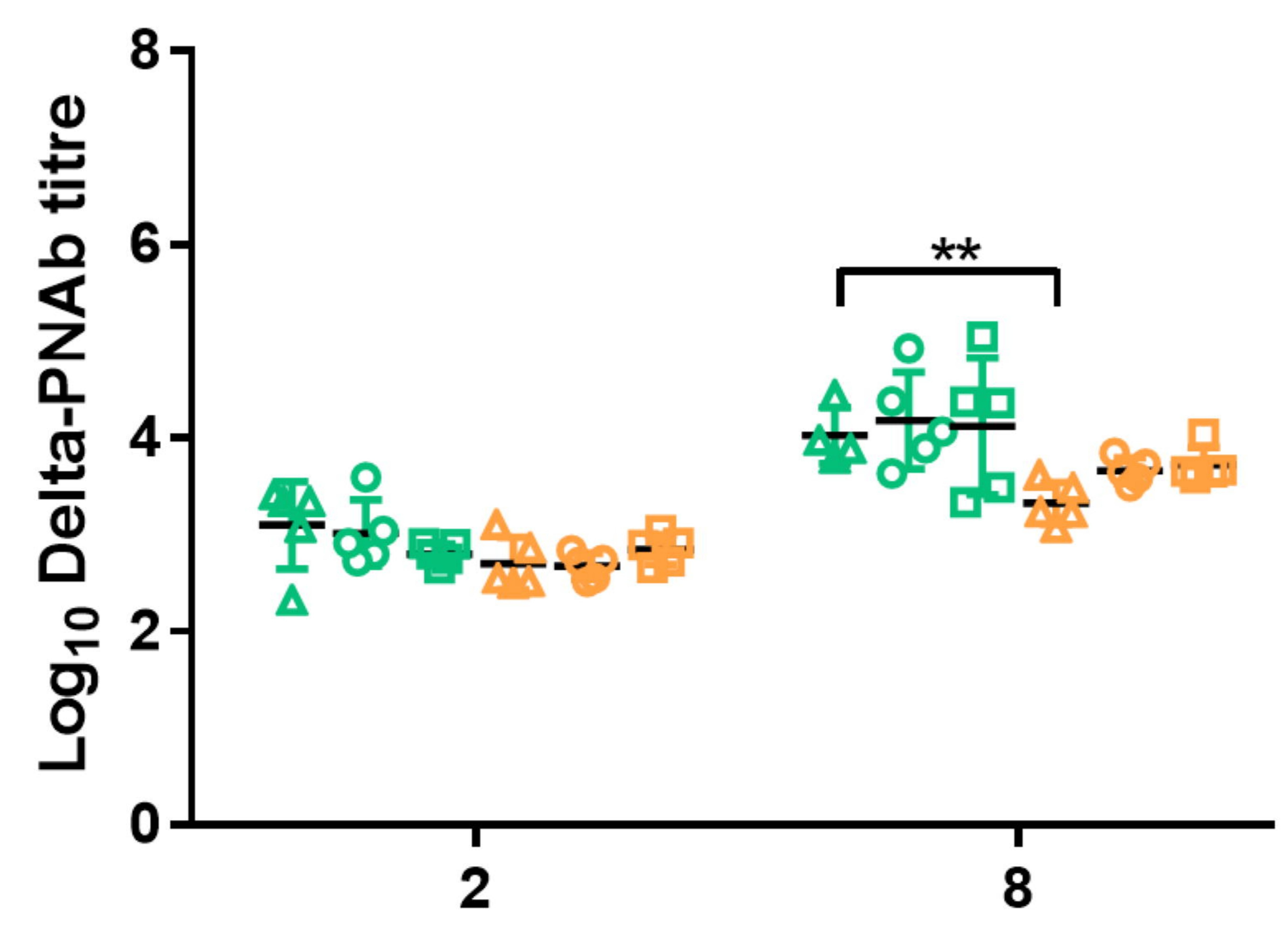

Weeks post vaccination

C

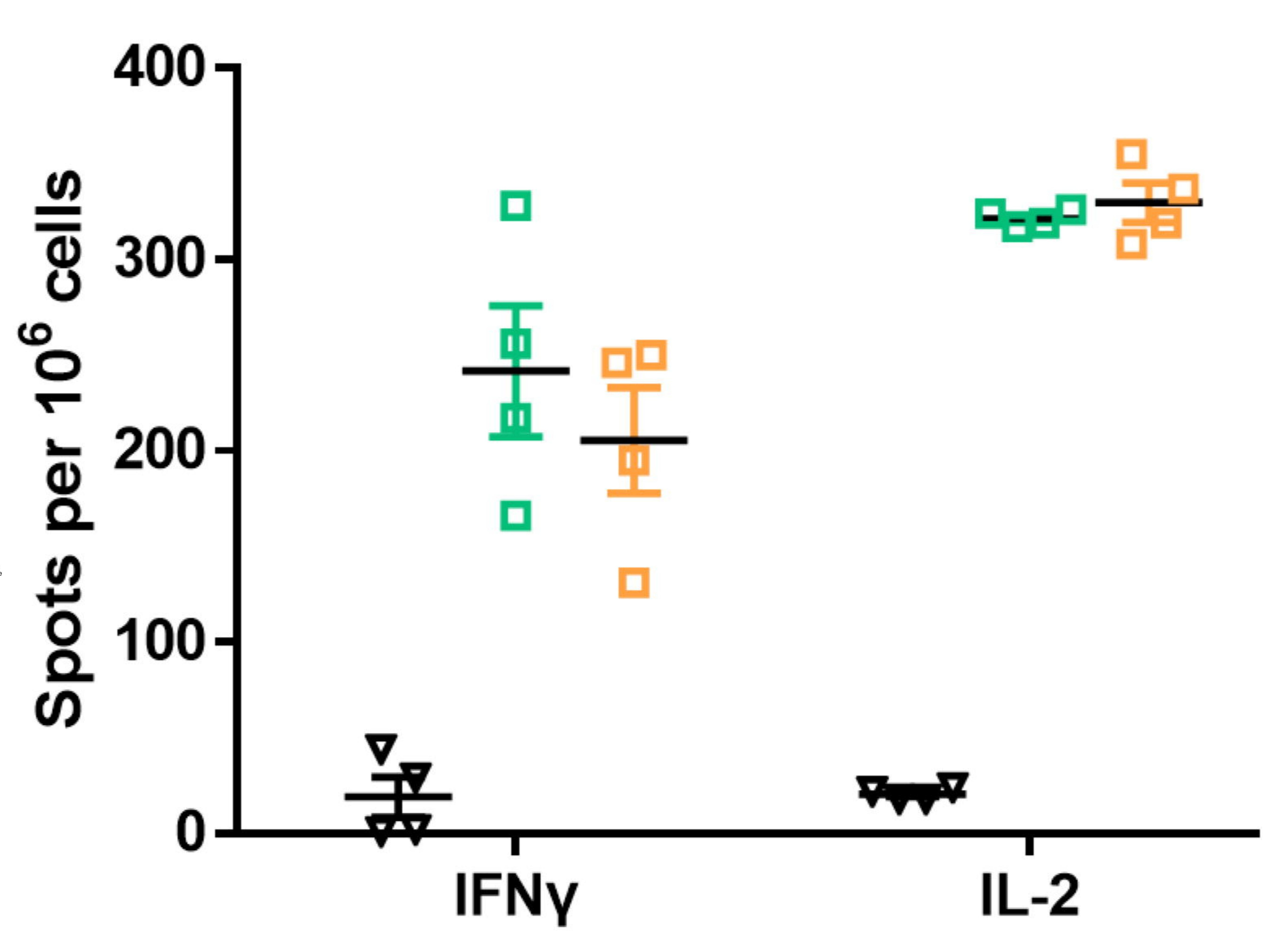

$\Delta \quad \mathrm{IN}-5 \times 10^{7} \mathrm{VP}$

- IN $-5 \times 10^{8} \mathrm{VP}$

- IN $-5 \times 10^{9} \mathrm{VP}$

IM- $5 \times 10^{7} \mathrm{VP}$

IM- $5 \times 10^{8} \mathrm{VP}$

IM- $5 \times 10^{9} \mathrm{VP}$

$\Delta \quad \mathrm{IN}-5 \times 10^{7} \mathrm{VP}$

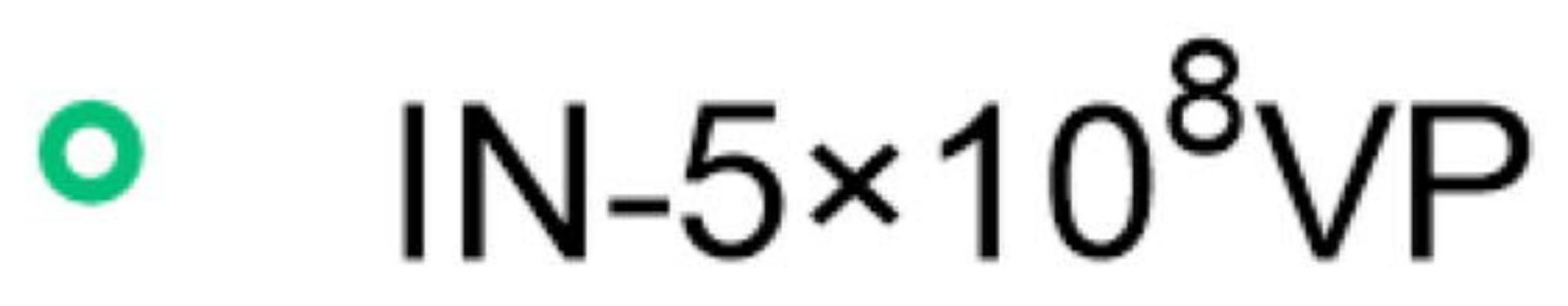

- IN- $5 \times 10^{9} \mathrm{VP}$

$\triangle \quad \mathrm{IM}-5 \times 10^{7} \mathrm{VP}$

- IM- $5 \times 10^{8} \mathrm{VP}$

- IM- $5 \times 10^{9} \mathrm{VP}$

$\nabla$ control vector

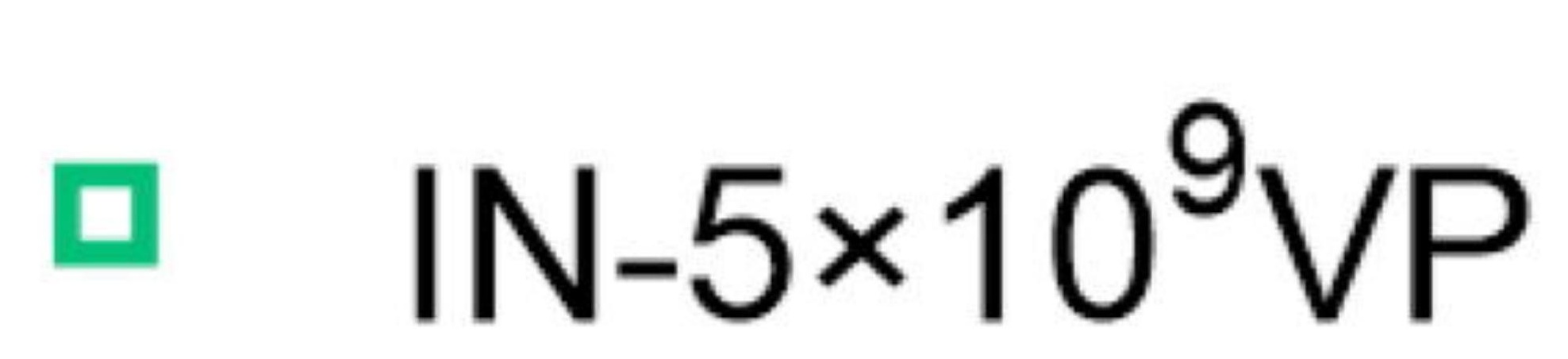

- IM- $5 \times 10^{9} \mathrm{VP}$
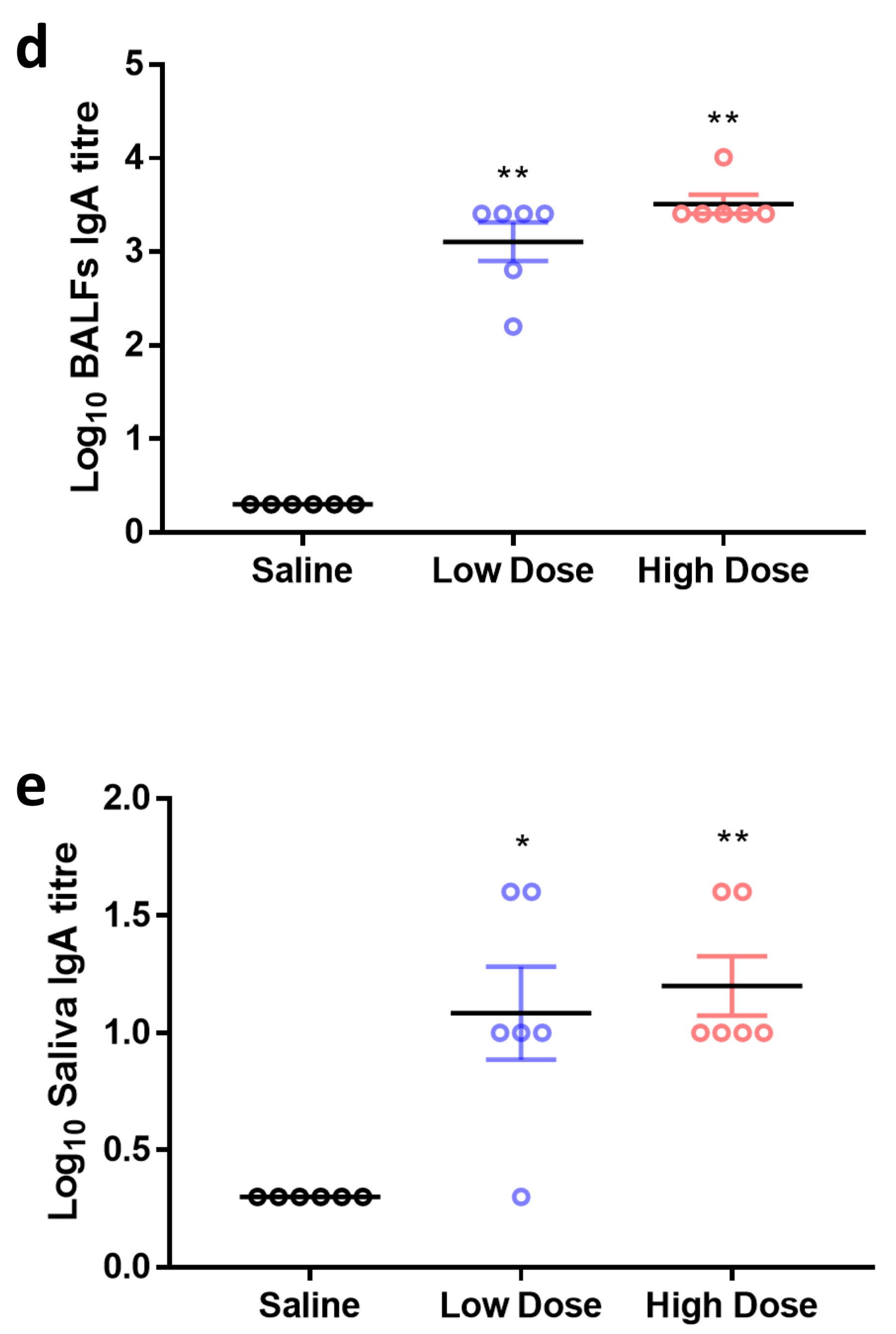

f

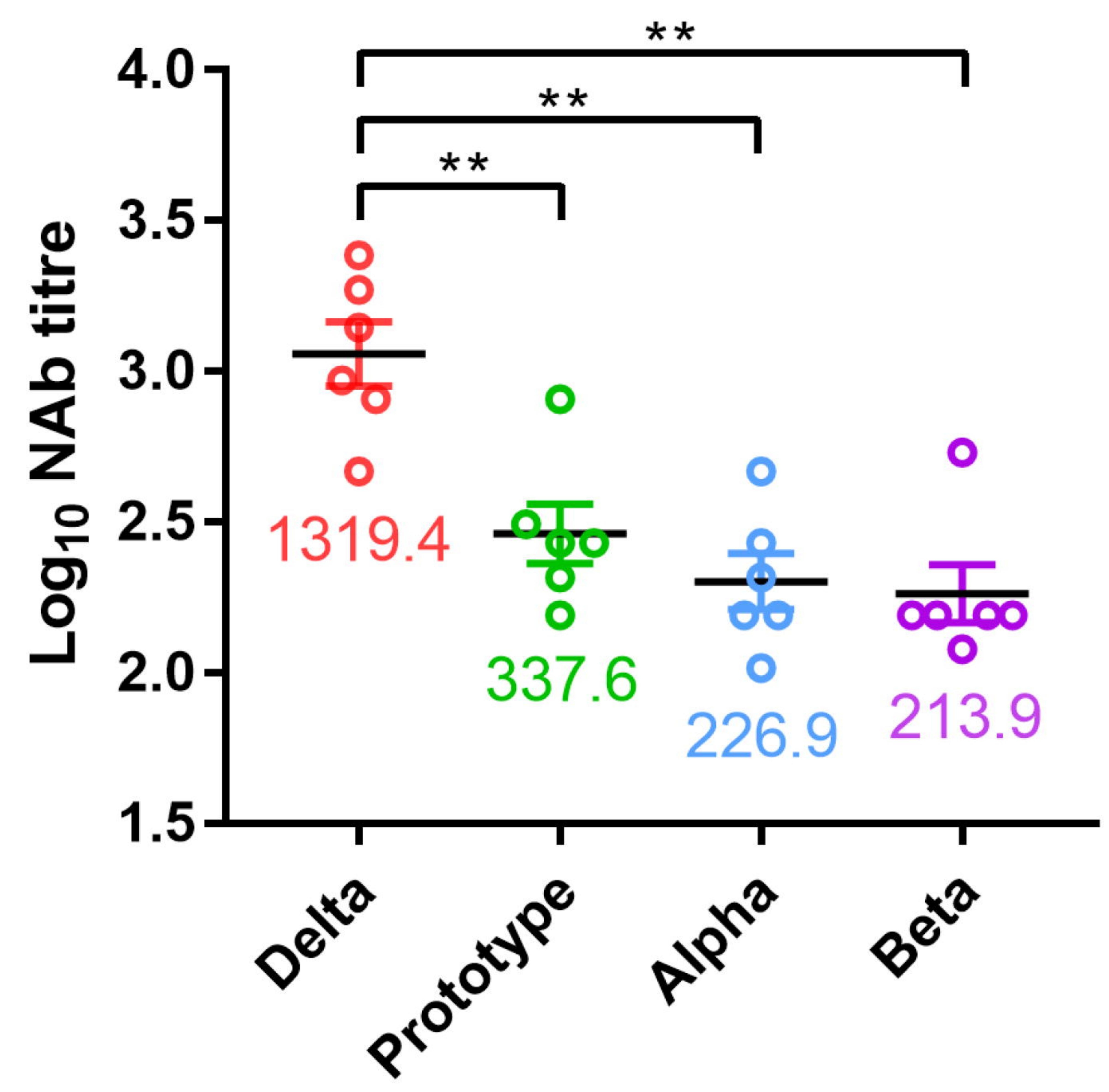


Fig.3

a

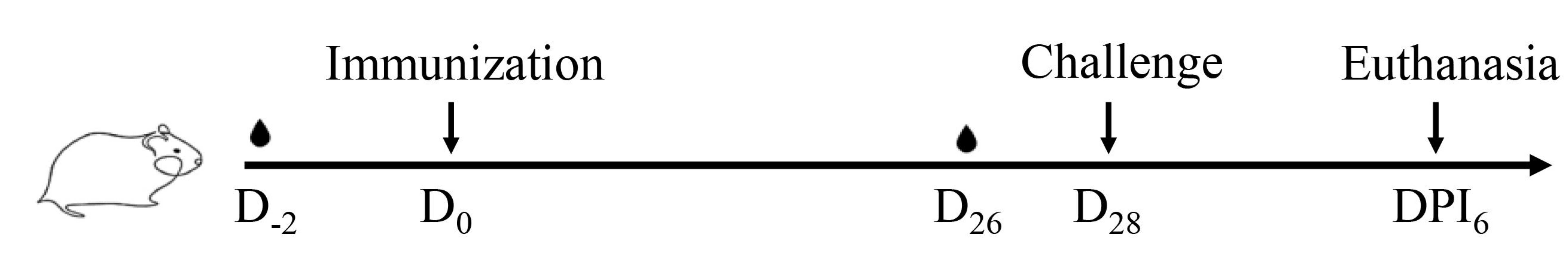

\begin{tabular}{|c|c|c|}
\hline & JS1-delta & Challenge \\
\hline Mock & -- & -- \\
\hline Saline & -- & + \\
\hline Low Dose & + & + \\
\hline High Dose & ++ & + \\
\hline
\end{tabular}

b

C
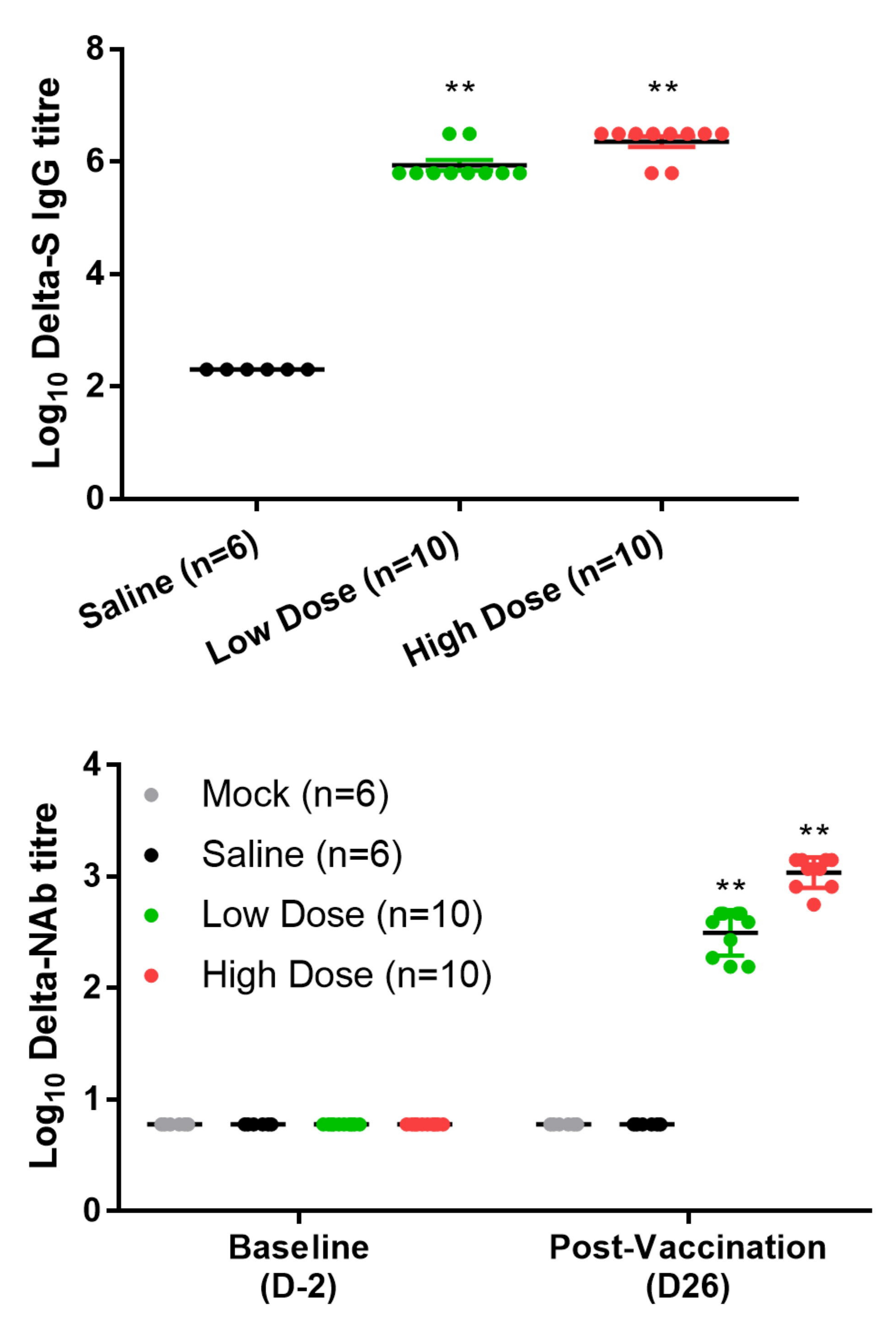

g

Mock

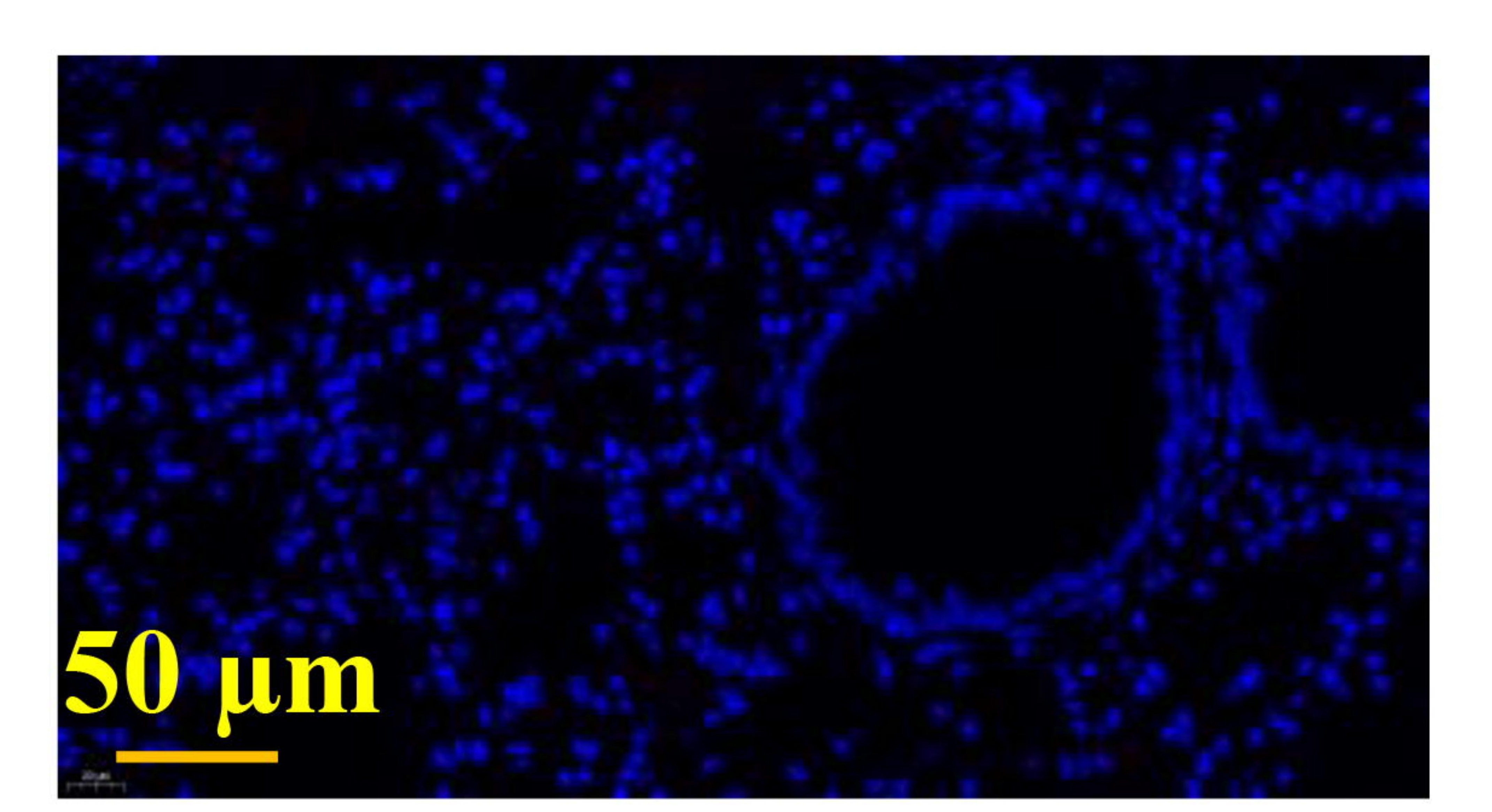

Saline

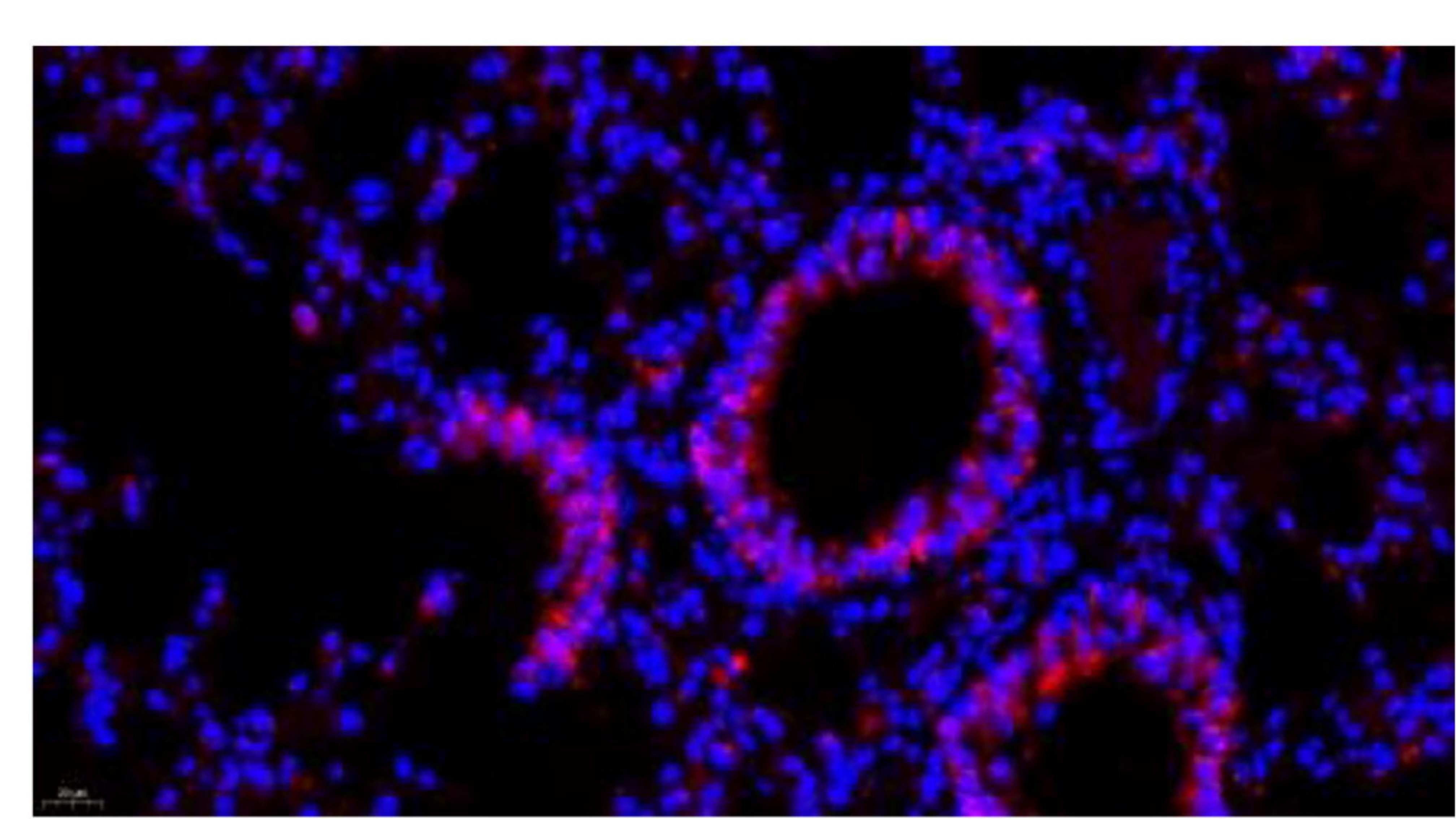

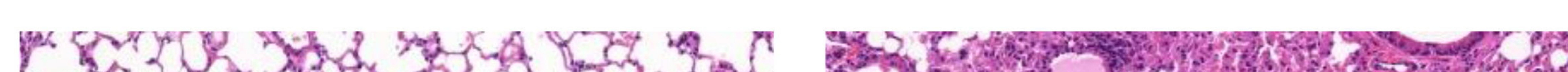

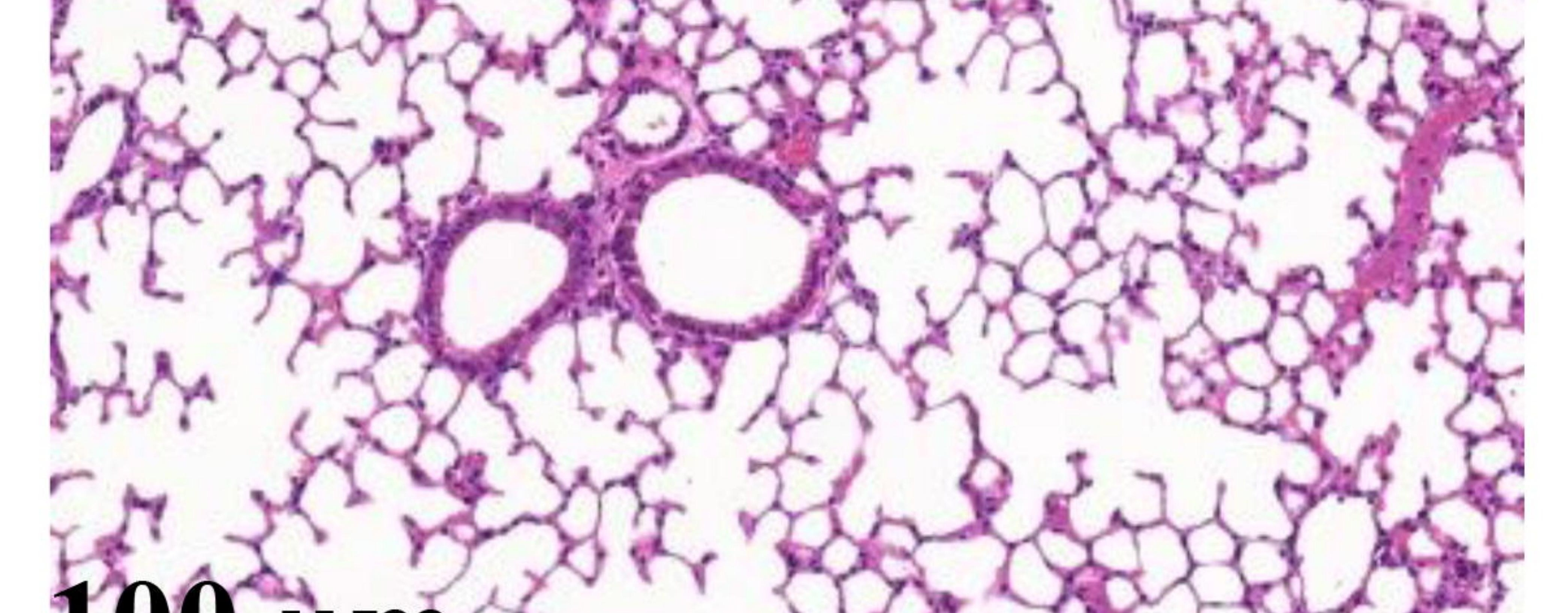
$100 \mathrm{~mm}$. d

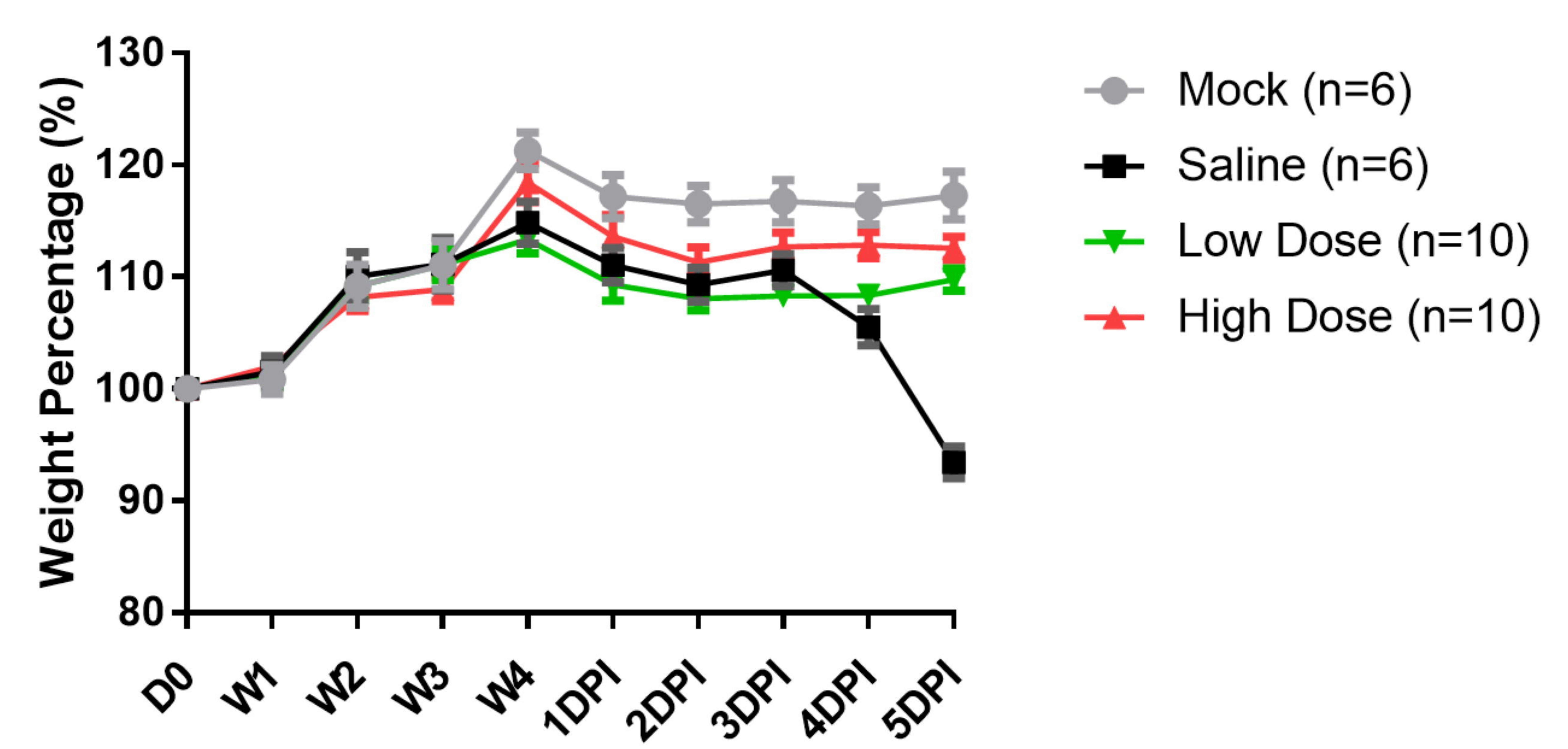

e

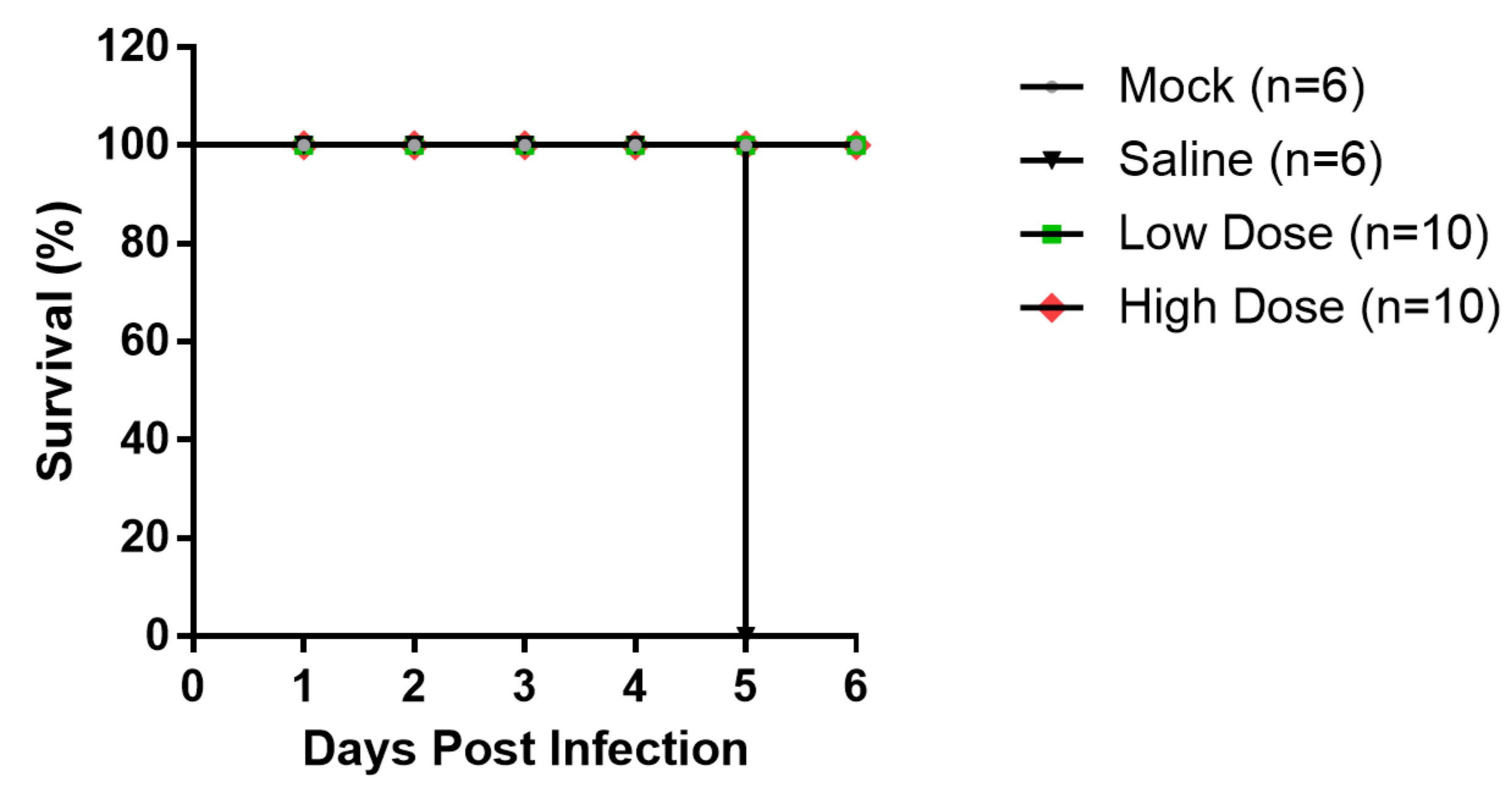

f

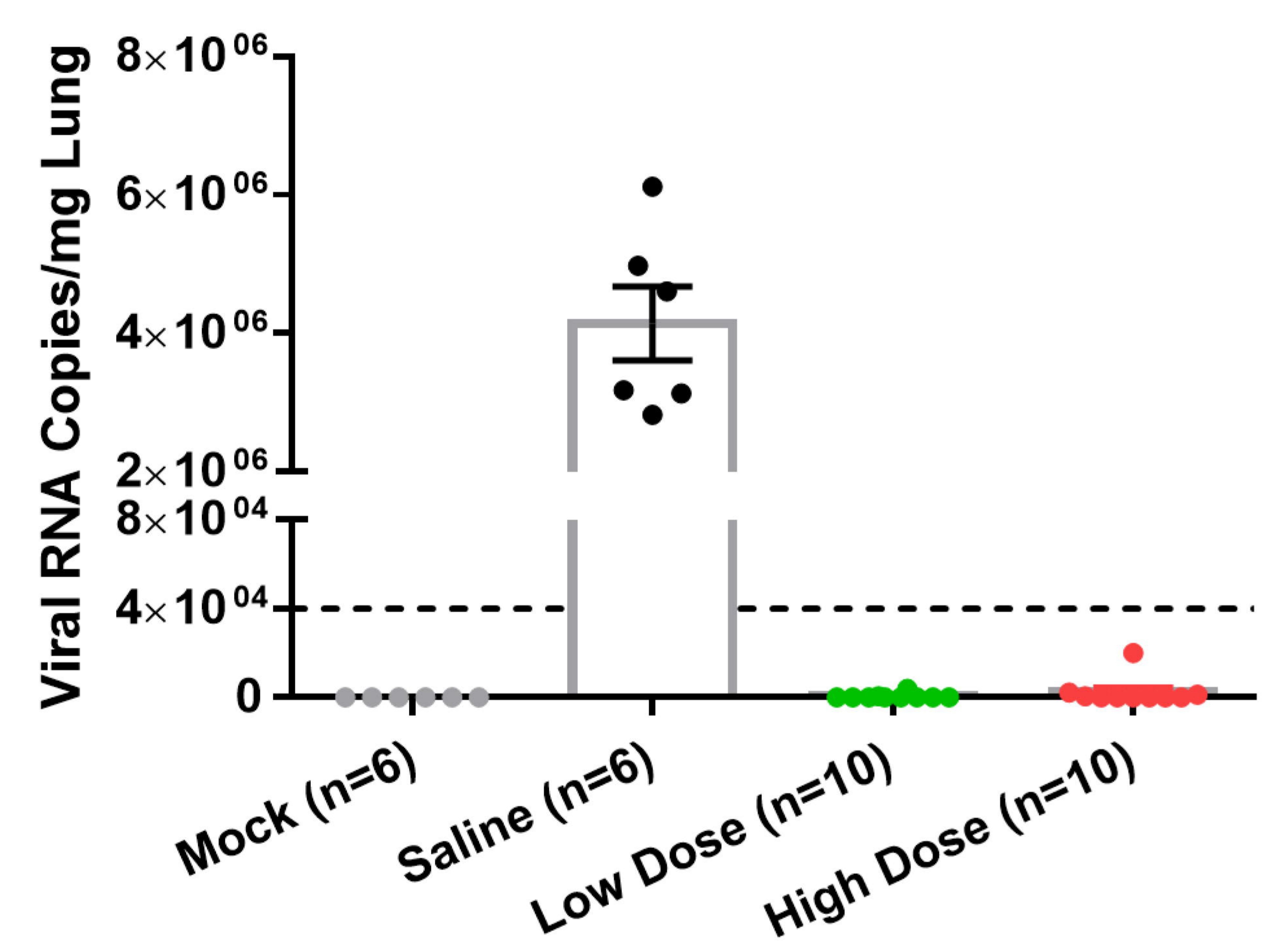

Low Dose

High Dose
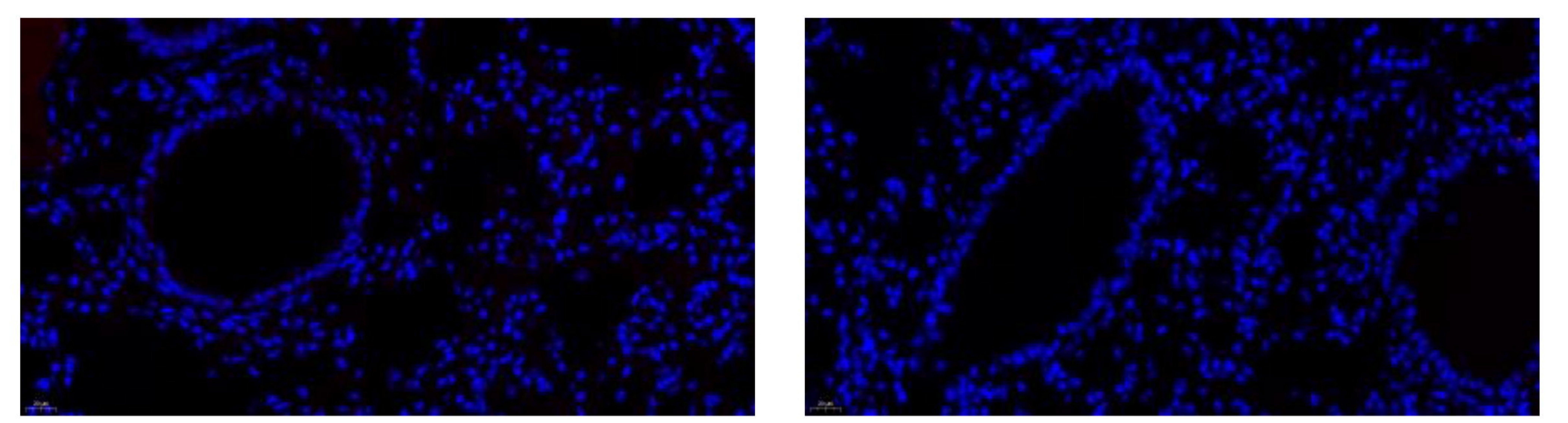

IF

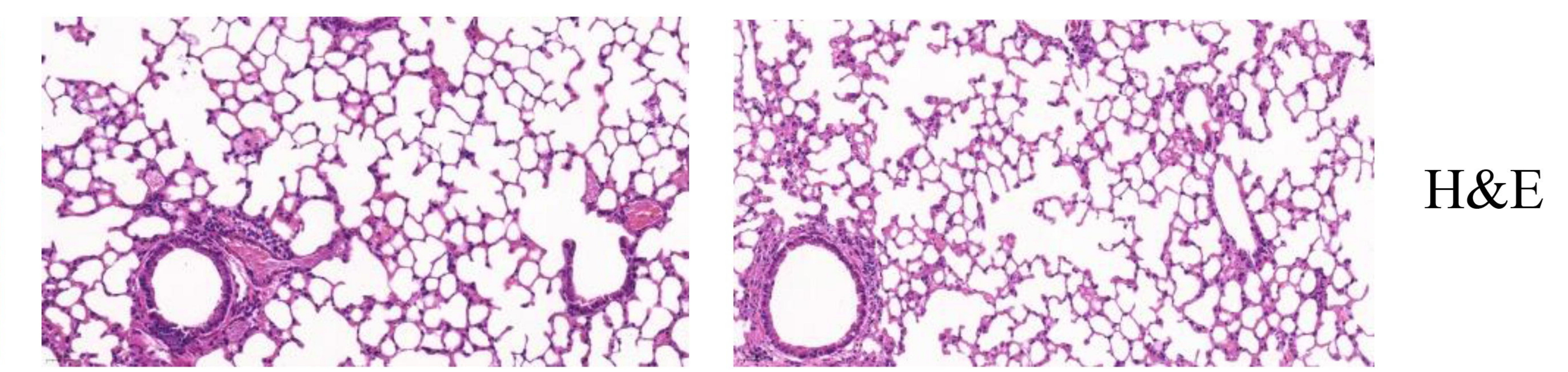

